\title{
Recurrent Multiple Squamous Cell Carcinomas on the Scalp in a Patient with Juvenile Dermatomyositis
}

\author{
Yota Sato Taku Fujimura Yumi Kambayashi Akira Tsukada \\ Takanori Hidaka Kayo Tanita Takahiro Haga Akira Hashimoto \\ Setsuya Aiba \\ Department of Dermatology, Tohoku University Graduate School of Medicine, \\ Sendai, Japan
}

\section{Keywords}

Cutaneous squamous cell carcinoma $\cdot$ IL-17/IL-17R · Signaling $\cdot$ Carcinogenesis

\begin{abstract}
Both long-term administration of immunosuppressive agents and chronic inflammatory conditions, such as autoimmune disease, could be risk factors for the development of cutaneous squamous cell carcinoma (CSCC). In this report, we present a case of recurrent multiple CSCC on the scalp in a patient with juvenile dermatomyositis who had been administered cyclosporine and Predonine since she was a 1-year-old infant. Interestingly, immunohistochemical staining revealed IL-17-producing cells adjacent to IL-17R-expressing atypical keratinocytes. Our present case suggested that IL-17/IL-17R signaling might contribute to the carcinogenesis of CSCC.

\section{Introduction}

Cutaneous squamous cell carcinoma (CSCC) is the second most common type of nonmelanoma skin cancer, and the risk factors for cSCC have been widely reported [1, 2]. Among them, the long-term administration of immunosuppressive agents has been reported as a 


\section{Case Reports in Oncology}

high risk factor for the development of cSCC, especially on sun-exposed areas of the skin [3]. On the other hand, chronic inflammatory conditions, such as autoimmune disease, could also be risk factors for the development of cSCC [4]. In this report, we present a case of recurrent multiple cSCC on the scalp in a patient with juvenile dermatomyositis who had been administered cyclosporine and prednisolone since she was a 1-year-old infant.

\section{Case Report}

A 33-year-old woman visited our outpatient clinic with an erosive nodule on the scalp. She had been treated for juvenile dermatomyositis and had been administered immunosuppressive agents such as cyclosporine and prednisolone since she was 1 year old. On her initial visit, physical examination revealed a dome-shaped, easy-to-bleed nodule on her parietal scalp (Fig. 1a). In addition, a wide-spreading scarring alopecia-like lesion was observed on the whole scalp. The size of the nodule was approximately $32 \mathrm{~mm}$ in diameter. Skin biopsy from the nodule revealed a dermal infiltration composed of atypical keratinocytes with dyskeratotic cytoplasm (Fig. 1b). Around the tumor mass, a dense infiltration of lymphocytes was observed. We diagnosed this patient as having a moderately differentiated SCC on the parietal scalp and excised the tumor with a $10-\mathrm{mm}$ margin. Four years after the initial excision, another red nodule developed in the left temporal region (Fig. 1c). Since the histological findings of the second tumor were similar to those of the first cutaneous SCC (Fig. 1d), we excised the tumor with a $10-\mathrm{mm}$ margin. Eleven months after the second surgical treatment, a keratotic lesion was apparent around the grafted area (Fig. 1e), and again we excised it with a 10-mm margin. The histological findings of the third tumor were similar to those of the first cutaneous SCC, which was a widely spread SCC in situ (Fig. 1f). Additionally, we performed a random biopsy on the patient's scalp, and there were no atypical cells - only homogenization of the collagen bundle with carcinosis.

Since recurrent multi-focal lesions were detected in this case, and since a previous report suggested that IL-17 plays a key role in cutaneous SCC carcinogenesis, we employed immunohistochemical staining for IL-17 as well as IL-17R in the lesional skin of each of the cutaneous SCC in our case. Immunohistochemical staining revealed that these atypical keratinocytes strongly expressed IL-17R (Fig. 2a, b), whereas normal keratinocytes in the marginal area of the tumor did not (Fig. 2c). In contrast, IL-17-producing cells were detected in the dermis of both the tumor area and the marginal area (Fig. 2d).

\section{Discussion}

Long-term administration of immunosuppressive agents can cause DNA damage and deviations in natural immune surveillance [3]. For example, the risk factors for nonmelanoma skin cancer, such as CSCC, in transplant recipients are related to the dosage and duration of the administration of immunosuppressive reagents [5]. Indeed, in the present case our patient had been administered cyclosporine for juvenile dermatomyositis since she was 1 year old and developed multiple recurrent cSCC on the scalp. Our present case also provides insights into the relationships between the immunological background of CSCC and carcinogenesis.

Concerning the proinflammatory cytokines, $\mathrm{Wu}$ et al. [6] recently reported the contribution of the IL-17 signaling pathway to the tumorigenesis of cSCC. They concluded that IL-17- 


\section{Case Reports in Oncology}

\begin{tabular}{l|l}
\hline Case Rep Oncol 2017;10:106-111 \\
\hline DOI: 10.1159/000456001 & $\begin{array}{l}\text { C 2017 The Author(s). Published by S. Karger AG, Basel } \\
\text { www.karger.com/cro }\end{array}$ \\
\hline
\end{tabular}

Sato et al.: Recurrent Multiple Squamous Cell Carcinomas on the Scalp in a Patient with Juvenile Dermatomyositis

induced p63-mediated TRAF4 expression, which drives the IL-17-dependent sustained activation of the TRAF4-ERK5 axis, leading to keratinocyte proliferation and tumor formation in cSCC [6]. In addition, several clinical reports have suggested possible relationships between IL-17 and the development of cSCC [7, 8]. For example, Mayama et al. [7] reported that severe bacterial infection might induce IL-17-producing cells and CD163+ tumor-associated macrophages (TAMs) in the lesional skin of cSCC arising from keratitis-ichthyosis-deafness (KID) syndrome, leading to the development of multiple cSCC. In another report, Tsukada et al. [8] reported a cSCC arising from recessive dystrophic epidermolysis bullosa, which possesses a substantial number of IL-17-producing cells and CD163+ TAMs. Since TAMs are a heterogeneous population of cells that produce specific chemokines by the stimulation of stromal factors [9], and chronic overproduction of IL-17 is generally observed in autoimmune diseases such as psoriasis, rheumatoid arthritis, and inflammatory bowel disease [10], TAMs in proinflammatory conditions might be stimulated by IL-17 or other proinflammatory cytokines to recruit IL-17-producing cells in the lesional skin of CSCC. Although the number of TAMs in conventional CSCC is higher than in keratoacanthoma [11], the function of TAMs is still mostly unknown. Therefore, further experiments will be needed to clarify the relationship between TAMs and IL-17-producing cells.

Based on the above findings, we employed immunohistochemical staining of IL-17 and IL-17R to investigate the tumor-loading factors in our present case, especially focusing on keratinocytes. As we expected, substantial numbers of IL-17-producing cells were observed adjacent to the tumor. Moreover, the expression of IL-17R was prominent on tumor cells but not on normal keratinocytes, suggesting the importance of IL-17R expression in the progression of cSCC. Although we still do not know the immunomodulatory factors that upregulate the IL-17R expression on tumor cells in CSCC, the findings of our present study suggest the significance of IL-17/IL-17R signaling in the development of cSCC.

\section{Statement of Ethics}

The patient gave written informed consent.

\section{Disclosure Statement}

The authors have no conflict of interest to declare.

\section{References}

1 Thompson AK, Kelley BF, Prokop LJ, Murad MH, Baum CL: Risk factors for cutaneous squamous cell carcinoma recurrence, metastasis, and disease-specific death: a systematic review and meta-analysis. JAMA Dermatol 2016;152:419-428.

2 Cheng J, Yan S: Prognostic variables in high-risk cutaneous squamous cell carcinoma: a review. J Cutan Pathol 2016;43:994-1004.

3 Kasiske BL, Snyder JJ, Gilbertson DT, Wang C: Cancer after kidney transplantation in the United States. Am J Transplant 2004;4:905-913.

-4 Drake AL, Walling HW: Variations in presentation of squamous cell carcinoma in situ (Bowen's disease) in immunocompromised patients. J Am Acad Dermatol 2008;59:68-71.

-5 Pini AM, Koch S, Schärer L, French LE, Läuchli S, Hofbauer GF: Eruptive keratoacanthoma following topical imiquimod for in situ squamous cell carcinoma of the skin in a renal transplant recipient. J Am Acad Dermatol 2008;59(suppl 5):S116-S117. 
Sato et al.: Recurrent Multiple Squamous Cell Carcinomas on the Scalp in a Patient with Juvenile Dermatomyositis

6 Wu L, Chen X, Zhao J, Martin B, Zepp JA, Ko JS, Gu C, Cai G, Ouyang W, Sen G, Stark GR, Su B, Vines CM, Tournier C, Hamilton TA, Vidimos A, Gastman B, Liu C, Li X: A novel IL-17 signaling pathway controlling keratinocyte proliferation and tumorigenesis via the TRAF4-ERK5 axis. J Exp Med 2015;212:15711587.

7 Mayama H, Fujimura T, Asano M, Kambayashi Y, Numata Y, Aiba S: Squamous cell carcinoma arising from keratitis-ichthyosis-deafness syndrome. Acta Derm Venereol 2013;93:583-584.

-8 Tsukada A, Fujimura T, Furudate S, Kambayashi Y, Numata Y, Haga T, Hashimoto A, Aiba S: Cutaneous squamous cell carcinoma developing from recessive dystrophic epidermolysis bullosa: a case report and an immunohistochemical study. Case Rep Dermatol 2012;4:197-201.

$\$ 9$ Fujimura T, Kakizaki A, Furudate S, Kambayashi Y, Aiba S: Tumor-associated macrophages in skin: how to treat their heterogeneity and plasticity. J Dermatol Sci 2016;83:167-173.

10 Golden JB, McCormick TS, Ward NL: IL-17 in psoriasis: implications for therapy and cardiovascular comorbidities. Cytokine 2013;62:195-201.

11 Kambayashi Y, Fujimura T, Aiba S: Comparison of immunosuppressive cells and immunomodulatory cells in kerartoacanthoma and invasive squamous cell carcinoma. Acta Derm Venereol 2013;93:663668. 


\section{Case Reports in Oncology}

\begin{tabular}{l|l}
\hline Case Rep Oncol 2017;10:106-111 \\
\hline DOI: 10.1159/000456001 & $\begin{array}{l}\text { (c) 2017 The Author(s). Published by S. Karger AG, Basel } \\
\text { www.karger.com/cro }\end{array}$ \\
\hline
\end{tabular}

Sato et al.: Recurrent Multiple Squamous Cell Carcinomas on the Scalp in a Patient with Juvenile Dermatomyositis
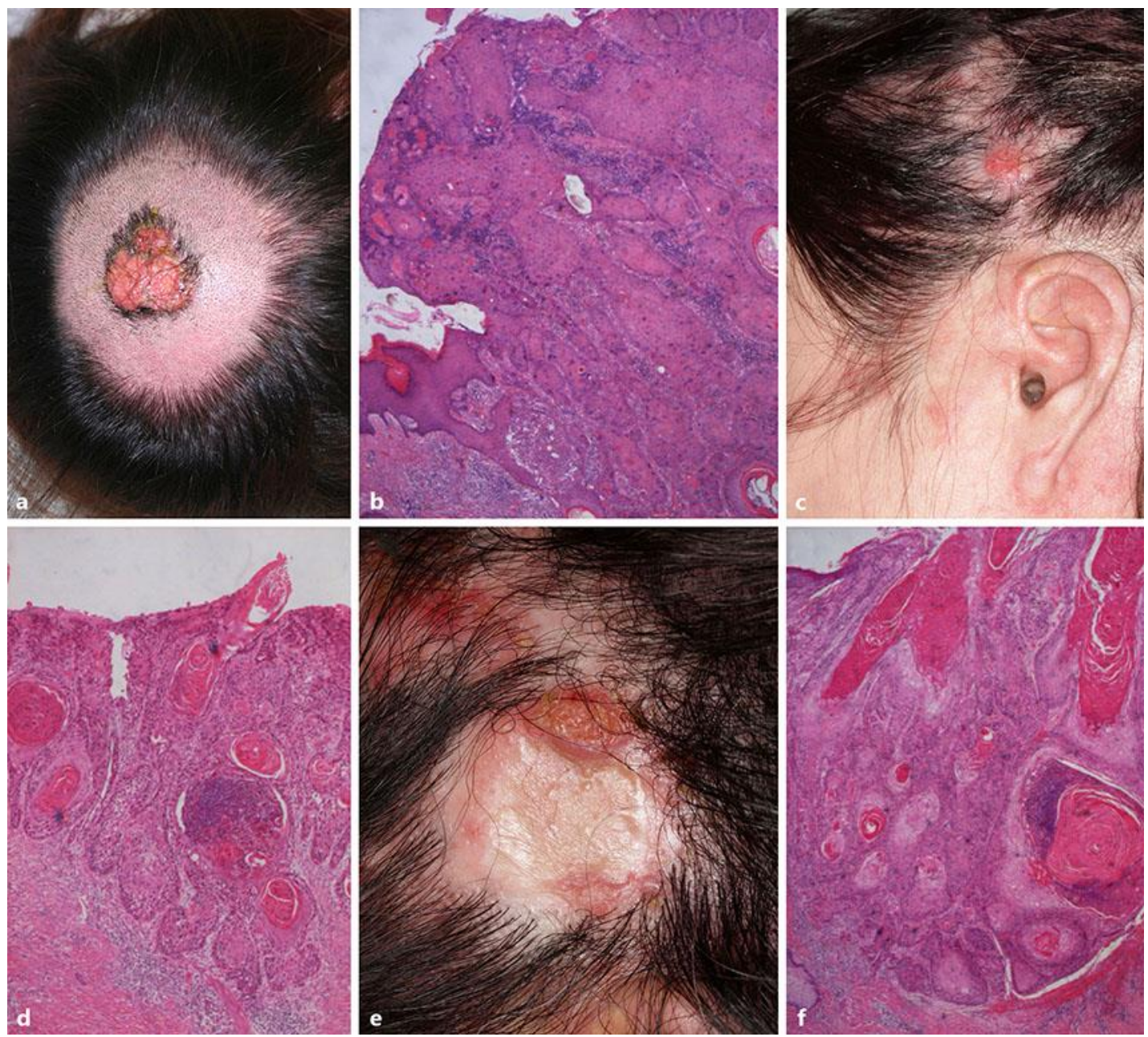

Fig. 1. a A dome-shaped, easy-to-bleed nodule on the parietal scalp. b Dermal infiltration of atypical keratinocytes with dyskeratotic cytoplasm. c Another red nodule that developed in the left temporal region. $\mathbf{d}$ Dermal infiltration of atypical keratinocytes with dyskeratotic cytoplasm. e Keratotic lesions were apparent around the grafted area. $\mathbf{f}$ Dermal infiltration of atypical keratinocytes with dyskeratotic cytoplasm. 


\section{Case Reports in Oncology}
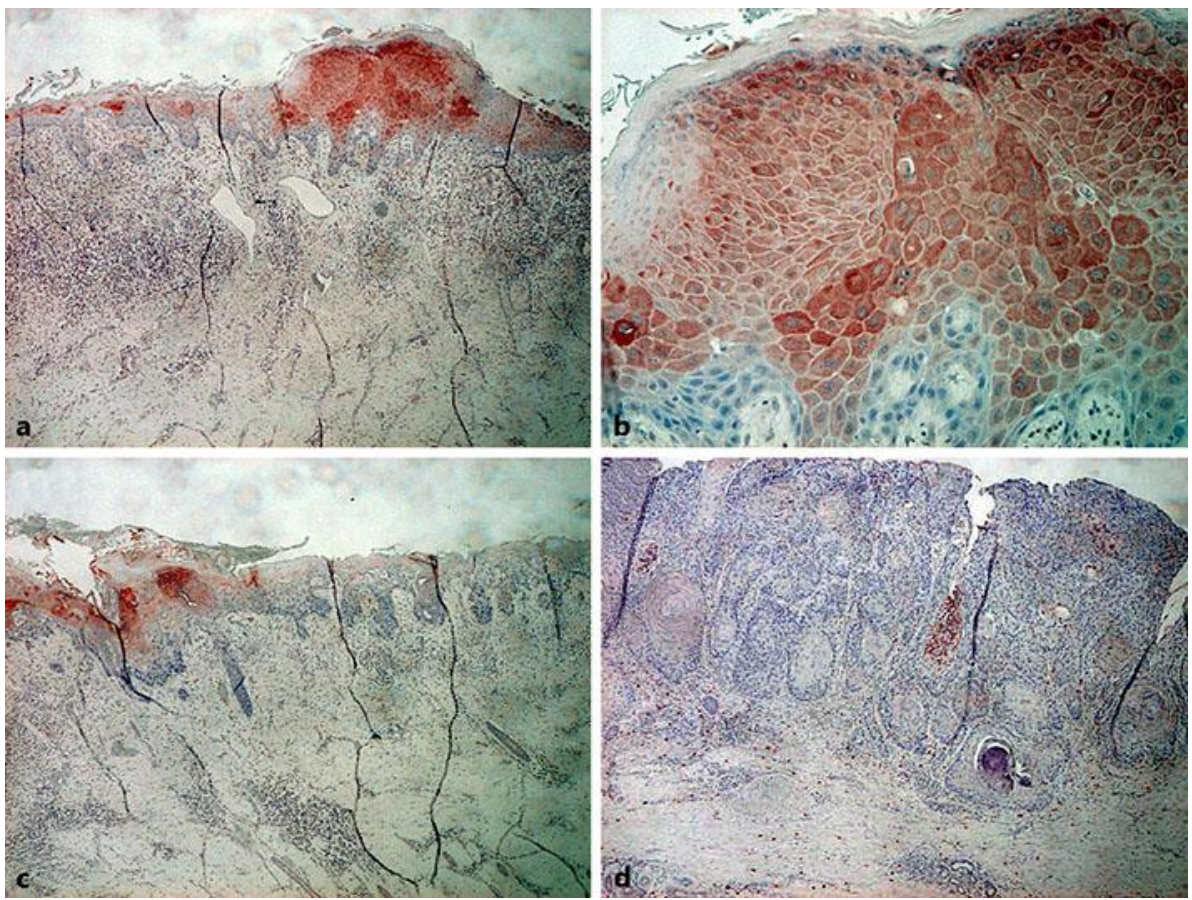

Fig. 2. Paraffin-embedded tissue samples from the tumor sites (a, b, d) or surgical margin (c) were deparaffinized and stained with anti-IL-17R antibody (a-c) and anti-IL-17 antibody (d). The sections were developed with Liquid Permanent Red. Original magnification, $\times 50$ (a, c), ×100 (d), ×200 (b). 